\title{
Existence, uniqueness and stability of positive solutions to a general sublinear elliptic systems
}

Boying $\mathrm{Wu}^{1}$ and Renhao Cui ${ }^{1,2^{*}}$

${ }^{*}$ Correspondence:

renhaocui@gmail.com

${ }^{1}$ Department of Mathematics,

Harbin Institute of Technology,

Harbin, Heilongjiang 150001,

P.R. China

${ }^{2}$ Y.Y. Tseng Functional Analysis Research Center and School of

Mathematical Sciences, Harbin

Normal University, Harbin,

Heilongjiang 150025, P.R. China

\begin{abstract}
In this paper, we make use of a new stability result and bifurcation theory to study the existence and uniqueness of positive solutions to semilinear elliptic systems with some general sublinear conditions. Moreover, we obtain the precise global bifurcation diagrams of the system in a single monotone solution curve.

MSC: $35 \mathrm{~J} 55 ; 35 \mathrm{~B} 32$
\end{abstract}

Keywords: semilinear elliptic systems; positive solution; stability; existence; uniqueness

\section{Introduction}

We consider positive solutions of a semilinear elliptic system with $n$ equations $(n \geq 2)$

$$
\begin{cases}\Delta u_{1}+\lambda f_{1}\left(u_{1}, u_{2}, \ldots, u_{n}\right)=0, & x \in \Omega, \\ \Delta u_{2}+\lambda f_{2}\left(u_{1}, u_{2}, \ldots, u_{n}\right)=0, & x \in \Omega, \\ \cdots & \\ \Delta u_{n}+\lambda f_{n}\left(u_{1}, u_{2}, \ldots, u_{n}\right)=0, & x \in \Omega, \\ u_{1}(x)=\cdots=u_{n}(x)=0, & x \in \partial \Omega,\end{cases}
$$

where $\lambda>0$ is a positive parameter, $\Omega$ is a bounded smooth domain in $\mathbf{R}^{N}$, and $f_{i}(i=$ $1,2, \ldots, n)$ are smooth real-valued functions defined on $\mathbf{R}_{+}^{n}=\left\{\left(u_{1}, u_{2}, \ldots, u_{n}\right) \in \mathbf{R}^{n}: u_{i}>\right.$ $0,1 \leq i \leq n\}$ satisfying the following.

Cooperativeness Define the Jacobian of the vector field $\left(f_{1}, \ldots, f_{n}\right)$ as

$$
H\left(u_{1}, \ldots, u_{n}\right)=\left(\begin{array}{ccc}
\frac{\partial f_{1}}{\partial u_{1}} & \cdots & \frac{\partial f_{1}}{\partial u_{n}} \\
\vdots & \ddots & \vdots \\
\frac{\partial f_{n}}{\partial u_{1}} & \cdots & \frac{\partial f_{n}}{\partial u_{n}}
\end{array}\right)=\left(\begin{array}{ccc}
f_{11}^{\prime} & \cdots & f_{1 n}^{\prime} \\
\vdots & \ddots & \vdots \\
f_{n 1}^{\prime} & \cdots & f_{n n}^{\prime}
\end{array}\right) .
$$

Then $\partial f_{i} / \partial u_{j} \geq 0(i \neq j)$ for $\left(u_{1}, \ldots, u_{n}\right) \in \mathbf{R}_{+}^{n}$.

The purpose of this paper is to study the existence, uniqueness and stability of positive solutions of such cooperative system (1.1) under certain conditions of $f_{i}$.

@ 2013 Wu and Cui; licensee Springer. This is an Open Access article distributed under the terms of the Creative Commons Attribution License (http://creativecommons.org/licenses/by/2.0), which permits unrestricted use, distribution, and reproduction in any medium, provided the original work is properly cited. 
The existence, uniqueness and stability of positive solutions to sublinear semilinear elliptic systems with two equations have been recently studied in [1-4]. The sublinear condition plays an important role. In this paper, we continue the effort in [3] to prove the stability of a positive solution to (1.1) under some reasonable sublinear conditions, and the stability implies the uniqueness of the positive solution. We also prove corresponding existence results using bifurcation theory and the continuation method. This is motivated by the existence study of exact multiplicity (and uniqueness) of positive solutions to the scalar semilinear elliptic equation:

$$
\begin{cases}\Delta u+\lambda f(u)=0, & x \in \Omega, \\ u(x)=0, & x \in \partial \Omega,\end{cases}
$$

starting from Korman et al. [5, 6]. In Ouyang and Shi [7, 8], their result classified the different global exact multiplicity of (1.3) for more general nonlinearity $f$. There are more results on the existence and uniqueness of solution to the semilinear cyclic elliptic system

$$
\begin{cases}\Delta u+\lambda f(v)=0, & x \in \Omega, \\ \Delta v+\lambda g(u)=0, & x \in \Omega, \\ u(x)=v(x)=0, & x \in \partial \Omega .\end{cases}
$$

The notation of sublinearity and superlinearity of the nonlinear vector field or the ones in higher dimension was considered in Sirakov [9]. Our definition of sublinear nonlinearity is quite different, and ours is similar to the one in Ouyang and Shi [8] for the scalar case. Dalmasso [10] obtained the existence and uniqueness result for a more special sublinear system, and it was extended by Shi and Shivaji [4]. The uniqueness of a positive solution for large $\lambda$ was proved in Hai $[11,12]$, Hai and Shivaji [13]. If $\Omega$ is a finite ball or the whole space, then the positive solutions of systems are radically symmetric and decreasing in radical direction by the result of Troy [14]. Hence the system can be converted into a system of ODEs. Several authors have taken that approach for the existence of the solutions, see Serrin and Zou [15, 16], and much success has been achieved for Lane-Emden systems. Using the scaling invariant in the Lane-Emden system, the uniqueness of the radial positive solution was shown in Dalmasso [10], Korman and Shi [17]. Cui et al. [18, 19] considered cyclic systems with three equations, and the uniqueness and existence of positive solutions were obtained. For the Lane-Emden systems with $n$ equations, Maniwa [20] obtained the uniqueness and existence of positive solutions to systems under the sublinear conditions.

We organize the rest of this paper in the following way. In Section 2, we recall the maximum principle and prove the main stability result. In Section 3, we use the stability result and bifurcation theory to prove the existence and uniqueness of a positive solution. We also obtain the precise global bifurcation diagrams of the system (the bifurcation diagram is a single monotone solution curve in all cases) and give some examples. In Section 4, we consider the similar question for merely Hölder continuous nonlinearities, and we use monotone methods for existence. We use $W^{2, p}(\Omega)$ and $W_{\text {loc }}^{2, p}(\Omega)$ for the standard Sobolev space, $C(\bar{\Omega})$ for the space of continuous functions defined on $\bar{\Omega}$, and $C_{0}(\bar{\Omega})=\{u \in C(\bar{\Omega}): u(x)=0, x \in \partial \Omega\}$. We use $N(L)$ and $R(L)$ to denote the null space and the range space of a linear operator $L$. 


\section{Stability and linearized equations}

In this section, we study the stability result about a positive solution. Let $U=\left(u_{1}, \ldots, u_{n}\right)$ be a solution of (1.1). We shall denote the partial derivative of $f_{i}(U)$ with respect to $u_{j}$ by $f_{i j}^{\prime}(U)$ or $f_{i j}^{\prime}$. The stability of $U$ is determined by the eigenvalue equation

$$
\begin{cases}\Delta \xi_{1}+\lambda f_{11}^{\prime} \xi_{1}+\lambda f_{12}^{\prime} \xi_{2}+\cdots+\lambda f_{1 n}^{\prime} \xi_{n}=-\mu \xi_{1}, & x \in \Omega, \\ \Delta \xi_{2}+\lambda f_{21}^{\prime} \xi_{1}+\lambda f_{22}^{\prime} \xi_{2}+\cdots+\lambda f_{2 n}^{\prime} \xi_{n}=-\mu \xi_{2}, & x \in \Omega, \\ \cdots & \\ \Delta \xi_{n}+\lambda f_{n 1}^{\prime} \xi_{1}+\lambda f_{n 2}^{\prime} \xi_{2}+\cdots+\lambda f_{n n}^{\prime} \xi_{n}=-\mu \xi_{n}, & x \in \Omega, \\ \xi_{1}(x)=\xi_{2}(x)=\cdots=\xi_{n}(x)=0, & x \in \partial \Omega,\end{cases}
$$

which can be written as

$$
L \mathbf{u}=H \mathbf{u}+\mu \mathbf{u},
$$

where

$$
\mathbf{u}=\left(\begin{array}{c}
\xi_{1} \\
\vdots \\
\xi_{n}
\end{array}\right), \quad L \mathbf{u}=\left(\begin{array}{c}
-\Delta \xi_{1} \\
\vdots \\
-\Delta \xi_{n}
\end{array}\right), \quad \text { and } \quad H=\left(\begin{array}{ccc}
f_{11}^{\prime} & \cdots & f_{1 n}^{\prime} \\
\vdots & \ddots & \vdots \\
f_{n 1}^{\prime} & \cdots & f_{n n}^{\prime}
\end{array}\right) .
$$

Definition 2.1 [16] An $n \times n$ matrix $A$ is reducible if for some permutation matrix $Q$,

$$
Q A Q^{T}=\left(\begin{array}{ll}
B & 0 \\
C & D
\end{array}\right)
$$

where $B$ and $C$ are square matrices and $Q^{T}$ is the transpose of $Q$. Otherwise, $A$ is irreducible.

Throughout this paper, $H$ is assumed to be irreducible, since if not the case, the linearized system (2.1) can be reduced to two subsystems with one being not coupled with the other. If we assume that $H$ is cooperative and irreducible, then the maximum principles hold for such systems. Before stating our results, we recall some known results as required.

Lemma 2.2 Let $X=\left[W_{\text {loc }}^{2, p}(\Omega) \cap C_{0}(\bar{\Omega})\right]^{n}$, and let $Y=\left[L^{p}(\Omega)\right]^{n}$ for $p>n$. Suppose that $L, H$ are given as in (2.3), and $H=\left(f_{i j}^{\prime}\right)$ is irreducible and satisfies $f_{i j}^{\prime} \geq 0(i \neq j)$ for $\left(u_{1}, \ldots, u_{n}\right) \in \mathbf{R}_{+}^{n}$. Then we have the following.

1. $\mu_{1}=\inf \{\mu \in \operatorname{spt}(L-H)\}$ is a real eigenvalue of $L-H$, where $\operatorname{spt}(L-H)$ is the spectrum of $L-H$.

2. For $\mu=\mu_{1}$, there exists a unique (up a constant multiple) eigenfunction $\mathbf{u}_{1} \in X$, and $\mathbf{u}_{1}>0$ in $\Omega$.

3. For $\mu<\mu_{1}$, the equation $L \mathbf{u}=H \mathbf{u}+\mu \mathbf{u}+\mathbf{f}$ is uniquely solvable for any $\mathbf{f} \in Y$, and $\mathbf{u}>0$ as long as $\mathbf{f} \geq 0$.

4. (Maximum principle) For $\mu \leq \mu_{1}$, suppose that $\mathbf{u} \in\left[W_{\text {loc }}^{2, p}(\Omega) \cap C(\bar{\Omega})\right]^{n}$ satisfies $L \mathbf{u} \geq H \mathbf{u}+\mu \mathbf{u}$ in $\Omega, \mathbf{u} \geq 0$ on $\partial \Omega$, then $\mathbf{u} \geq 0$ in $\Omega$. 
5. If there exists $\mathbf{u} \in\left[W_{\mathrm{loc}}^{2, p}(\Omega) \cap C(\bar{\Omega})\right]^{n}$ satisfying $L \mathbf{u} \geq H \mathbf{u}$ in $\Omega, \mathbf{u} \geq 0$ on $\partial \Omega$, and either $\mathbf{u} \equiv 0$ on $\partial \Omega$ or $L \mathbf{u} \not \equiv H \mathbf{u}$ in $\Omega$, then $\mu_{1}>0$.

For the result and proofs, see Sweers [21], Proposition 3.1 and Theorem 1.1. Moreover, from a standard compactness argument, there are countably many eigenvalues $\left\{\mu_{i}\right\}$ of $L-H$, and $\left|\mu_{i}-\mu_{1}\right| \rightarrow \infty$ as $i \rightarrow \infty$. We notice that $\mu_{i}$ is not necessarily real-valued. We call a solution $U$ stable if $\mu_{1}>0$; and otherwise, it is unstable $\left(\mu_{1} \leq 0\right)$.

For our purpose, in this section, we also need to consider the adjoint operator of $L-H$. Let the transpose matrix of the Jacobian be

$$
H^{T}=\left(\begin{array}{ccc}
f_{11}^{\prime} & \cdots & f_{n 1}^{\prime} \\
\vdots & \ddots & \vdots \\
f_{1 n}^{\prime} & \cdots & f_{n n}^{\prime}
\end{array}\right) .
$$

Then, evidently, the results in Lemma 2.2 also hold for the eigenvalue problem

$$
L \mathbf{u}^{*}=H^{T} \mathbf{u}^{*}+\mu \mathbf{u}^{*}
$$

which is

$$
\begin{cases}\Delta \xi_{1}^{*}+\lambda f_{11}^{\prime} \xi_{1}^{*}+\lambda f_{21}^{\prime} \xi_{2}^{*}+\cdots+\lambda f_{n 1}^{\prime} \xi_{n}^{*}=-\mu \xi_{1}^{*}, & x \in \Omega, \\ \Delta \xi_{2}^{*}+\lambda f_{12}^{\prime} \xi_{1}^{*}+\lambda f_{22}^{\prime} \xi_{2}^{*}+\cdots+\lambda f_{n 2}^{\prime} \xi_{n}^{*}=-\mu \xi_{2}^{*}, & x \in \Omega, \\ \cdots & \\ \Delta \xi_{n}^{*}+\lambda f_{1 n}^{\prime} \xi_{1}^{*}+\lambda f_{2 n}^{\prime} \xi_{2}^{*}+\cdots+\lambda f_{n n}^{\prime} \xi_{n}^{*}=-\mu \xi_{n}^{*}, & x \in \Omega, \\ \xi_{1}^{*}(x)=\xi_{2}^{*}(x)=\cdots=\xi_{n}^{*}(x)=0, & x \in \partial \Omega,\end{cases}
$$

where $\mathbf{u}^{*}=\left(\xi_{1}^{*}, \xi_{2}^{*}, \ldots, \xi_{n}^{*}\right)^{T}$. It is easy to verify that $L-H^{T}$ is the adjoint operator of $L-H$, while both are considered as operators defined on subspaces of $\left[L^{2}(\Omega)\right]^{n}$. By using the well-known functional analytic techniques (see $[21,22])$, one can show the following.

Lemma 2.3 Let $X, Y, L$ and $H$ be the same as in Lemma 2.2. Then the principal eigenvalue $\mu_{1}$ of $L-H$ is also a real eigenvalue of $L-H^{T}, \mu_{1}=\inf \left\{\mu \in \operatorname{spt}\left(L-H^{T}\right)\right\}$, and for $\mu=\mu_{1}$, there exists a unique eigenfunction $\mathbf{u}_{1}^{*} \in\left[W_{\operatorname{loc}}^{2, p}(\Omega) \cap C_{0}(\bar{\Omega})\right]^{n}$ of $L-H^{T}$ (up a constant multiple), and $\mathbf{u}_{1}^{*}>0$ in $\Omega$.

Cui et al. [3] obtained the stability result of a positive solution for the system with two equations. We give the following stability result about a positive solution of (1.1).

Theorem 2.4 Suppose that $U=\left(u_{1}, \ldots, u_{n}\right)$ is a positive solution of $(1.1), f_{i}$ is cooperative and $H=\left(f_{i j}^{\prime}\right)$ is irreducible, then $U$ is stable if $f_{i}$ satisfies one of the following conditions: for any $U \in \mathbf{R}_{+}^{n}$,

$$
f_{i}(U)>\sum_{j=1}^{n} f_{i j}^{\prime}(U) u_{j}, \quad i=1, \ldots, n ; \quad \text { or }
$$


$\left(\mathrm{H}_{2}\right)$

$$
f_{i}(U)>\sum_{j=1}^{n} f_{j i}^{\prime}(U) u_{j}, \quad i=1, \ldots, n .
$$

Proof Let $U=\left(u_{1}, \ldots, u_{n}\right)$ be a positive solution of $(1.1)$, and let $\left(\mu_{1}, \mathbf{u}^{*}\right)$ be the corresponding principal eigen-pair of (2.6) such that $\xi_{i}^{*}>0$ in $\Omega$ for any $1 \leq i \leq n$.

We assume that $f_{i}$ satisfies $\left(\mathrm{H}_{1}\right)$. Multiplying the system (1.1) by $\mathbf{u}^{*}$, the system (2.6) by $U$, integrating over $\Omega$ and subtracting, we obtain

$$
\begin{aligned}
\mu_{1} \int_{\Omega} \sum_{i=1}^{n} u_{i} \xi_{i}^{*} d x & =\lambda \int_{\Omega} \sum_{i=1}^{n} f_{i} \xi_{i}^{*} d x-\lambda \int_{\Omega} \sum_{i=1}^{n} \xi_{i}^{*} \sum_{j=1}^{n} f_{i j}^{\prime} u_{j} d x \\
& =\lambda \int_{\Omega} \sum_{i=1}^{n} \xi_{i}^{*}\left(f_{i}-\sum_{j=1}^{n} f_{i j}^{\prime} u_{j}\right) d x .
\end{aligned}
$$

Hence $\mu_{1}>0$ if $\left(\mathrm{H}_{1}\right)$ is satisfied.

Similar to the proof above, let $\left(\mu_{1}, \mathbf{u}\right)$ be the corresponding principal eigen-pair of (2.1) such that $\xi_{i}>0$ in $\Omega$ for any $i=1, \ldots, n$. We assume that $f_{i}$ satisfies $\left(\mathrm{H}_{2}\right)$. Multiplying the system (1.1) by $\mathbf{u}$, the system (2.1) by $U$, integrating over $\Omega$ and subtracting, we can get

$$
\begin{aligned}
\mu_{1} \int_{\Omega} \sum_{i=1}^{n} u_{i} \xi_{i} d x & =\lambda \int_{\Omega} \sum_{i=1}^{n} f_{i} \xi_{i} d x-\lambda \int_{\Omega} \sum_{i=1}^{n} \xi_{i} \sum_{j=1}^{n} f_{j i}^{\prime} u_{j} d x \\
& =\lambda \int_{\Omega} \sum_{i=1}^{n} \xi_{i}\left(f_{i}-\sum_{j=1}^{n} f_{j i}^{\prime} u_{j}\right) d x .
\end{aligned}
$$

Hence $\mu_{1}>0$ if $\left(\mathrm{H}_{2}\right)$ is satisfied.

On the other hand, the same proof also implies the following instability result under the opposite condition of $\left(\mathrm{H}_{1}\right)$ and $\left(\mathrm{H}_{2}\right)$.

Theorem 2.5 Suppose that $U=\left(u_{1}, \ldots, u_{n}\right)$ is a positive solution of (1.1), $f_{i}$ is cooperative and $H^{T}=\left(f_{j i}^{\prime}\right)$ is irreducible, then $U$ is unstable if $f_{i}$ satisfies one of the following conditions: for any $U \in \mathbf{R}_{+}^{n}$,

$\left(\mathrm{H}_{1}^{\prime}\right)$

$$
f_{i}(U)<\sum_{j=1}^{n} f_{i j}^{\prime}(U) u_{j}, \quad i=1, \ldots, n ; \quad \text { or }
$$

$\left(\mathrm{H}_{2}^{\prime}\right)$

$$
f_{i}(U)<\sum_{j=1}^{n} f_{j i}^{\prime}(U) u_{j}, \quad i=1, \ldots, n
$$




\section{Remark 2.6}

1. In [8], for positive solutions of the scalar equation

$$
\Delta u+\lambda h(u)=0, \quad x \in \Omega, \quad u(x)=0, \quad x \in \partial \Omega,
$$

the function $h(u)$ is called a sublinear function if $h(u)>u h^{\prime}(u)$, and it is superlinear if $h(u)<u h^{\prime}(u)$. It was proved in Proposition 3.14 of [8] that a positive solution $u$ is stable if $h$ is sublinear, and $u$ is unstable if $h$ is superlinear. Now our conclusions, Theorems 2.4 and 2.5, are generalizations of the corresponding results. The condition $\left(\mathrm{H}_{i}\right)$ for $i=1,2$ is the generalization of sublinearity (or superlinearity) to $n$-variable vector fields.

2. The conditions $\left(\mathrm{H}_{1}\right)$ and $\left(\mathrm{H}_{2}\right)$ can be written in a vector form $F(U)>H(U) U$ and $F(U)>H^{T}(U) U$, where $F(U)=\left(f_{1}(U), \ldots, f_{n}(U)\right)^{T}, U=\left(u_{1}, \ldots, u_{n}\right)^{T}$, and $H$ is the original Jacobian matrix of the vector field $\left(f_{1}(U), \ldots, f_{n}(U)\right)$, and $H^{T}$ is the transpose matrix of the Jacobian $H$. The condition $\left(\mathrm{H}_{1}\right)$ is clearly more natural as the conditions for $f_{i}$ are separate. Hence the sublinearity can be defined for a single $n$-variable function. The condition $\left(\mathrm{H}_{2}\right)$ is defined for the whole vector field $\left(f_{1}(U), \ldots, f_{n}(U)\right)$.

3. If a solution $U$ is stable, then it is necessarily a non-degenerate solution. That is, any eigenvalue $\mu_{i}$ of (2.1) has a positive real part. But when a solution is proved to be unstable, it can be a degenerate one with zero or pure imaginary eigenvalues.

\section{Existence and uniqueness}

In this section, we consider the uniqueness and existence of positive solutions for the following problem:

$$
\begin{cases}\Delta u_{1}+\lambda\left(g_{11}\left(u_{1}\right)+g_{12}\left(u_{2}\right)+\cdots+g_{1 n}\left(u_{n}\right)\right)=0, & x \in \Omega, \\ \Delta u_{2}+\lambda\left(g_{21}\left(u_{1}\right)+g_{22}\left(u_{2}\right)+\cdots+g_{2 n}\left(u_{n}\right)\right)=0, & x \in \Omega, \\ \cdots & \\ \Delta u_{n}+\lambda\left(g_{n 1}\left(u_{1}\right)+g_{n 2}\left(u_{2}\right)+\cdots+g_{n n}\left(u_{n}\right)\right)=0, & x \in \Omega, \\ u_{1}(x)=u_{2}(x)=\cdots=u_{n}(x)=0, & x \in \partial \Omega .\end{cases}
$$

Suppose that each $g_{i j}(x)(i, j=1,2, \ldots, n)$ is a smooth real function defined on $\mathbf{R}_{+}$satisfying

$\left(\mathrm{A}_{1}\right) g_{i j}(0) \geq 0$

$\left(\mathrm{A}_{2}\right) g_{i j}^{\prime}(x) \geq 0,\left(g_{i j}(x) / x\right)^{\prime} \leq 0$, for all $x \geq 0$.

The Perron-Frobenius theorem plays a critical role in our main result.

Lemma 3.1 (Perron-Frobenius theorem: strong form [23, Theorem 5.3.1]) Let $n \times n$ matrix $A$ be a nonnegative irreducible matrix. Then $\rho(A)$ is a simple eigenvalue of $A$, associated to a positive eigenvector, where $\rho(A)$ denotes the spectral radius of $A$. Moreover, $\rho(A)>0$.

Here let $\left(\lambda_{1}, \varphi_{1}\right)$ be the principal eigen-pair of

$$
-\Delta \varphi=\lambda \varphi, \quad x \in \Omega, \quad \varphi(x)=0, \quad x \in \partial \Omega
$$


such that $\varphi_{1}(x)>0$ in $\Omega$ and $\left\|\varphi_{1}\right\|_{\infty}=1$. We have the following existence and uniqueness result for this sublinear problem.

Theorem 3.2 Assume that each of $g_{i j}(x)$ satisfies $\left(\mathrm{A}_{1}\right),\left(\mathrm{A}_{2}\right)$ and

$\left(\mathrm{A}_{3}\right) \lim _{x \rightarrow \infty} \frac{g_{i j}(x)}{x}=0$.

1. If at least one of $g_{i j}(0)(i=1,2, \ldots, n)$ is positive and matrix $G=\left(g_{i j}(0)\right)$ is irreducible, then (3.1) has a unique positive solution $U(\lambda)=\left(u_{1}(\lambda), u_{2}(\lambda), \ldots, u_{n}(\lambda)\right)$ for all $\lambda>0$;

2. If $g_{i j}(0)=0, g_{i j}^{\prime}(0) \geq 0$ for each $i, j=1,2, \ldots, n$ and matrix $G^{\prime}=\left(g_{i j}^{\prime}(0)\right)$ is irreducible, then for some $\lambda_{*}>0$, (3.1) has no positive solution when $\lambda \leq \lambda_{*}$, and (3.1) has a unique positive solution $U(\lambda)=\left(u_{1}(\lambda), u_{2}(\lambda), \ldots, u_{n}(\lambda)\right)$ for $\lambda>\lambda_{*}$.

Moreover, $\left\{\left(\lambda, u_{1}(\lambda), u_{2}(\lambda), \ldots, u_{n}(\lambda)\right): \lambda>\lambda_{*}\right\}$ (in the first case, we assume $\lambda_{*}=0$ ) is a smooth curve so that $u_{i}(\lambda)$ is strictly increasing in $\lambda$, and $u_{i}(\lambda) \rightarrow 0$ as $\lambda \rightarrow \lambda_{*}^{+}$.

Proof Our proof follows that of Theorem 6.1 in [4]. Firstly, we extend $g_{i j}$ to be defined on $\mathbf{R}$ and they are continuously differentiable on $\mathbf{R}$. From $\left(\mathrm{A}_{2}\right), g_{i j}(x)>g_{i j}^{\prime}(x) x$ implies that $\sum_{j=1}^{n} g_{i j}\left(u_{j}\right)>\sum_{j=1}^{n} g_{i j}^{\prime}\left(u_{j}\right) u_{j}$, so $f_{i}(U)=f_{i}\left(u_{1}, \ldots, u_{n}\right)=\sum_{j=1}^{n} g_{i j}\left(u_{j}\right)$ satisfies $\left(\mathrm{H}_{1}\right)$. Hence from Theorem 2.4, any positive solution of (3.1) is stable.

Let us define

$$
F(\lambda, U)=\left(\begin{array}{c}
\Delta u_{1}+\lambda\left[g_{11}\left(u_{1}\right)+g_{12}\left(u_{2}\right)+\cdots+g_{1 n}\left(u_{n}\right)\right] \\
\Delta u_{2}+\lambda\left[g_{21}\left(u_{1}\right)+g_{22}\left(u_{2}\right)+\cdots+g_{2 n}\left(u_{n}\right)\right] \\
\cdots \\
\Delta u_{n}+\lambda\left[g_{n 1}\left(u_{1}\right)+g_{n 2}\left(u_{2}\right)+\cdots+g_{n n}\left(u_{n}\right)\right]
\end{array}\right)
$$

where $\lambda \in \mathbf{R}$ and $u_{1}, u_{2}, \ldots, u_{n} \in C_{0}^{2, \alpha}(\bar{\Omega})$. Here $g_{i j}$ are at least $C^{1}$, then $F: \mathbf{R} \times X \rightarrow Y$ is continuously differentiable, where $X=\left[C_{0}^{2, \alpha}(\bar{\Omega})\right]^{n}$ and $Y=\left[C^{\alpha}(\bar{\Omega})\right]^{n}$. For weak solutions $U=\left(u_{1}, \ldots, u_{n}\right)$, one can also consider $X=\left[W^{2, p}(\Omega) \cap W_{0}^{1, p}(\Omega)\right]^{n}$ and $Y=\left[L^{p}(\Omega)\right]^{n}$ where $p>1$ is properly chosen.

It is easy to see that $(\lambda, U)=(0,0, \ldots, 0)$ is a solution of (3.1). We apply the implicit function theorem at $(\lambda, U)=(0,0, \ldots, 0)$. The Fréchet derivative of $F$ is given by

$$
F_{U}(\lambda, U)\left(\begin{array}{c}
\phi_{1} \\
\phi_{2} \\
\vdots \\
\phi_{n}
\end{array}\right)=\left(\begin{array}{c}
\Delta \phi_{1}+\lambda\left[g_{11}^{\prime}\left(u_{1}\right) \phi_{1}+g_{12}^{\prime}\left(u_{2}\right) \phi_{2}+\cdots+g_{1 n}^{\prime}\left(u_{n}\right) \phi_{n}\right] \\
\Delta \phi_{2}+\lambda\left[g_{21}^{\prime}\left(u_{1}\right) \phi_{1}+g_{22}^{\prime}\left(u_{2}\right) \phi_{2}+\cdots+g_{2 n}^{\prime}\left(u_{n}\right) \phi_{n}\right] \\
\cdots \\
\Delta \phi_{n}+\lambda\left[g_{n 1}^{\prime}\left(u_{1}\right) \phi_{1}+g_{n 2}^{\prime}\left(u_{2}\right) \phi_{2}+\cdots+g_{n n}^{\prime}\left(u_{n}\right) \phi_{n}\right]
\end{array}\right) .
$$

Then $F_{U}(0,0, \ldots, 0)\left(\phi_{1}, \ldots, \phi_{n}\right)^{\mathrm{T}}=\left(\Delta \phi_{1}, \ldots, \Delta \phi_{n}\right)^{\mathrm{T}}$ is an isomorphism from $X$ to $Y$, and the implicit function theorem implies that $F(\lambda, U)=0$ has a unique solution $(\lambda, U(\lambda))$ for $\lambda \in(0, \delta)$ for some small $\delta>0$, and $\left(u_{1}^{\prime}(0), \ldots, u_{n}^{\prime}(0)\right)$ is the unique solution of

$$
\begin{cases}\Delta \phi_{1}+g_{11}(0)+g_{12}(0)+\cdots+g_{1 n}(0)=0, & x \in \Omega, \\ \Delta \phi_{2}+g_{21}(0)+g_{22}(0)+\cdots+g_{2 n}(0)=0, & x \in \Omega, \\ \cdots & \\ \Delta \phi_{n}+g_{n 1}(0)+g_{n 2}(0)+\cdots+g_{n n}(0)=0, & x \in \Omega, \\ \phi_{1}(x)=\cdots=\phi_{n}(x)=0, & x \in \partial \Omega .\end{cases}
$$


Therefore,

$$
\left\{\begin{array}{l}
u_{1}^{\prime}(0)=\left(g_{11}(0)+g_{12}(0)+\cdots+g_{1 n}(0)\right) e \\
u_{2}^{\prime}(0)=\left(g_{21}(0)+g_{22}(0)+\cdots+g_{2 n}(0)\right) e \\
\cdots \\
u_{n}^{\prime}(0)=\left(g_{n 1}(0)+g_{n 2}(0)+\cdots+g_{n n}(0)\right) e,
\end{array}\right.
$$

where $e$ is the unique positive solution of

$$
\Delta e+1=0, \quad x \in \Omega, \quad e(x)=0, \quad x \in \partial \Omega .
$$

If $\sum_{j=1}^{n} g_{i j}(0)>0$ for any $i=1,2, \ldots, n$, then $U(\lambda)=\left(u_{1}(\lambda), \ldots, u_{n}(\lambda)\right)$ is positive for $\lambda \epsilon$ $(0, \delta)$. If there exists $i$ such that $g_{i 1}(0)+g_{i 2}(0)+\cdots+g_{i n}(0)>0$, then $u_{i}(\lambda)>0$ for $\lambda \in(0, \delta)$. For $k \neq i, \Delta u_{k}(\lambda)=-\lambda\left[g_{k 1}(\lambda)+\cdots+g_{k n}(\lambda)\right]$ and $g_{i j}$ is positive, hence $u_{k}(\lambda)>0$ as well.

Next we assume that $g_{i j}(0)=0$ and $g_{i j}^{\prime}(0)>0(i, j=1, \ldots, n)$. The linearized operator is

$$
\begin{aligned}
F_{U}(\lambda, 0, \ldots, 0)\left(\begin{array}{c}
\Phi_{1} \\
\Phi_{2} \\
\vdots \\
\Phi_{n}
\end{array}\right) & =\left(\begin{array}{c}
\Delta \Phi_{1}+\lambda\left[g_{11}^{\prime}(0) \Phi_{1}+g_{12}^{\prime}(0) \Phi_{2}+\cdots+g_{1 n}^{\prime}(0) \Phi_{n}\right] \\
\Delta \Phi_{2}+\lambda\left[g_{21}^{\prime}(0) \Phi_{1}+g_{22}^{\prime}(0) \Phi_{2}+\cdots+g_{2 n}^{\prime}(0) \Phi_{n}\right] \\
\cdots \\
\Delta \Phi_{n}+\lambda\left[g_{n 1}^{\prime}(0) \Phi_{1}+g_{n 2}^{\prime}(0) \Phi_{2}+\cdots+g_{n n}^{\prime}(0) \Phi_{n}\right]
\end{array}\right) \\
& =\left(\begin{array}{c}
\Delta \Phi_{1} \\
\Delta \Phi_{2} \\
\vdots \\
\Delta \Phi_{n}
\end{array}\right)+\lambda\left(\begin{array}{cccc}
g_{11}^{\prime}(0) & g_{12}^{\prime}(0) & \cdots & g_{1 n}^{\prime}(0) \\
g_{21}^{\prime}(0) & g_{22}^{\prime}(0) & \cdots & g_{2 n}^{\prime}(0) \\
\vdots & \vdots & \ddots & \vdots \\
g_{n 1}^{\prime}(0) & g_{n 2}^{\prime}(0) & \cdots & g_{n n}^{\prime}(0)
\end{array}\right)\left(\begin{array}{c}
\Phi_{1} \\
\Phi_{2} \\
\vdots \\
\Phi_{n}
\end{array}\right) \\
& =\left(\begin{array}{c}
\Delta \Phi_{1} \\
\Delta \Phi_{2} \\
\vdots \\
\Delta \Phi_{n}
\end{array}\right)+\lambda J\left(\begin{array}{c}
\Phi_{1} \\
\Phi_{2} \\
\vdots \\
\Phi_{n}
\end{array}\right),
\end{aligned}
$$

where

$$
J=\left(\begin{array}{ccc}
g_{11}^{\prime}(0) & \cdots & g_{1 n}^{\prime}(0) \\
\vdots & \ddots & \vdots \\
g_{n 1}^{\prime}(0) & \cdots & g_{n n}^{\prime}(0)
\end{array}\right)
$$

Since $g_{i j}^{\prime}(0)>0$, all entries of matrix $J$ are positive. Therefore, by using Lemma 3.1, there exist a positive principal eigenvalue $\chi_{J}$ and the corresponding eigenvector $\left(k_{1}, k_{2}, \ldots, k_{n}\right)^{\mathrm{T}}$ of $J$ for some $k_{i}>0$ such that $\left(k_{1} \varphi_{1}, k_{2} \varphi_{1}, \ldots, k_{n} \varphi_{1}\right)^{\mathrm{T}}$ is a positive eigenvector of $F_{\left(u_{1}, \ldots, u_{n}\right)}\left(\lambda_{*}, 0\right.$, $\ldots, 0)$, where $\lambda_{*}=\lambda_{1} / \chi_{J}$. Similarly, $J^{\mathrm{T}}$ has the same principal eigenvalue $\chi_{J}$, and the corresponding eigenvector $\left(k_{1}^{*} \varphi_{1}, k_{2}^{*} \varphi_{1}, \ldots, k_{n}^{*} \varphi_{1}\right)^{\mathrm{T}}$, where $k_{i}^{*}(i=1, \ldots, n)$ is a positive constant.

Hence when $\lambda=\lambda_{*}=\lambda_{1} / \chi_{J}, F_{U}(\lambda, 0, \ldots, 0)$ is not invertible and $\lambda=\lambda_{*}$ is a potential bifurcation point. More precisely, the null space $N\left(F_{U}\left(\lambda_{*}, 0, \ldots, 0\right)\right)=\operatorname{span}\left\{\left(k_{1} \varphi_{1}, \ldots, k_{n} \varphi_{n}\right)\right\}$ is one-dimensional. 
Suppose that $\left(h_{1}, \ldots, h_{n}\right)^{\mathrm{T}} \in R\left(F_{U}\left(\lambda_{*}, 0, \ldots, 0\right)\right)$, then there exist $\left(\psi_{1}, \ldots, \psi_{n}\right) \in X$ such that

$$
\begin{aligned}
& F_{U}\left(\lambda_{*}, 0, \ldots, 0\right)\left(\begin{array}{c}
\psi_{1} \\
\vdots \\
\psi_{n}
\end{array}\right) \\
& =\left(\begin{array}{c}
\Delta \psi_{1} \\
\vdots \\
\Delta \psi_{n}
\end{array}\right)+\lambda_{*}\left(\begin{array}{ccc}
g_{11}^{\prime}(0) & \cdots & g_{1 n}^{\prime}(0) \\
\ldots & \ddots & \ldots \\
g_{n 1}^{\prime}(0) & \cdots & g_{n n}^{\prime}(0)
\end{array}\right)\left(\begin{array}{c}
\psi_{1} \\
\vdots \\
\psi_{n}
\end{array}\right)=\left(\begin{array}{c}
h_{1} \\
\vdots \\
h_{n}
\end{array}\right) .
\end{aligned}
$$

Consider the adjoint eigenvalue equation

$$
\left(\begin{array}{c}
\Delta \psi_{1}^{*} \\
\vdots \\
\Delta \psi_{n}^{*}
\end{array}\right)+\lambda_{*} J^{\mathrm{T}}\left(\begin{array}{c}
\psi_{1}^{*} \\
\vdots \\
\psi_{n}^{*}
\end{array}\right)=\left(\begin{array}{c}
\Delta \psi_{1}^{*} \\
\vdots \\
\Delta \psi_{n}^{*}
\end{array}\right)+\lambda_{*}\left(\begin{array}{ccc}
g_{11}^{\prime}(0) & \cdots & g_{n 1}^{\prime}(0) \\
\ldots & \ddots & \cdots \\
g_{1 n}^{\prime}(0) & \cdots & g_{n n}^{\prime}(0)
\end{array}\right)\left(\begin{array}{c}
\psi_{1}^{*} \\
\vdots \\
\psi_{n}^{*}
\end{array}\right)=0,
$$

where $\left(\psi_{1}^{*}, \ldots, \psi_{n}^{*}\right)^{\mathrm{T}}=\left(k_{1}^{*} \varphi_{1}, \ldots, k_{n}^{*} \varphi_{n}\right)^{\mathrm{T}}$.

Multiplying the system $(3.8)$ by $\left(\psi_{1}^{*}, \ldots, \psi_{n}^{*}\right)$, the system (3.9) by $\left(\psi_{1}, \ldots, \psi_{n}\right)$, integrating over $\Omega$ and subtracting, we get

$$
\int_{\Omega}\left(h_{1} \psi_{1}^{*}+\cdots+h_{n} \psi_{n}^{*}\right) d x=\int_{\Omega}\left(k_{1}^{*} h_{1} \varphi_{1}+\cdots+k_{n}^{*} h_{n} \varphi_{1}\right) d x=0 .
$$

Hence $\left(h_{1}, \ldots, h_{n}\right)^{\mathrm{T}} \in R\left(F_{U}\left(\lambda_{*}, 0, \ldots, 0\right)\right)$ if and only if (3.9) holds, which implies that the codimension of $R\left(F_{U}\left(\lambda_{*}, 0, \ldots, 0\right)\right)$ is one.

Next we verify that $F_{\lambda U}\left(\lambda_{*}, 0, \ldots, 0\right)\left[k_{1} \varphi_{1}, \ldots, k_{n} \varphi_{1}\right]^{\mathrm{T}} \notin R\left(F_{U}\left(\lambda_{*}, 0, \ldots, 0\right)\right)$. Indeed,

$$
F_{\lambda U}\left(\lambda_{*}, 0, \ldots, 0\right)\left(\begin{array}{c}
k_{1} \varphi_{1} \\
\vdots \\
k_{n} \varphi_{1}
\end{array}\right)=J\left(\begin{array}{c}
k_{1} \\
\vdots \\
k_{n}
\end{array}\right) \varphi_{1}=\chi_{J}\left(\begin{array}{c}
k_{1} \\
\vdots \\
k_{n}
\end{array}\right)
$$

But this contradicts with

$$
0=\chi_{J} \int_{\Omega}\left(k_{1} k_{1}^{*}+\cdots+k_{n} k_{n}^{*}\right) \varphi_{1}^{2} d x>0
$$

Hence

$$
F_{\lambda U}\left(\lambda_{*}, 0, \ldots, 0\right)\left[k_{1} \varphi_{1}, \ldots, k_{n} \varphi_{1}\right]^{\mathrm{T}} \notin R\left(F_{U}\left(\lambda_{*}, 0, \ldots, 0\right)\right) .
$$

By using a bifurcation from a simple eigenvalue theorem of Crandall-Rabinowitz [24], we conclude that $\left(\lambda_{*}, 0, \ldots, 0\right)$ is a bifurcation point. The nontrivial solutions of $F\left(\lambda, u_{1}, \ldots\right.$, $\left.u_{n}\right)=(0, \ldots, 0)$ near $\left(\lambda_{*}, 0, \ldots, 0\right)$ are in the form of $\left\{\left(\lambda(s), u_{1}(s), \ldots, u_{n}(s)\right)\right\}$ for $s \in(-\delta, \delta)$, where $u_{i}(s)=k_{i} s \varphi_{1}+o(s)(i=1, \ldots, n)$. From the stability of positive solutions, each positive solution is non-degenerate. 
Next we claim that (1.1) has no positive solution when $\lambda \leq \lambda_{*}$. Let $\left(u_{1}, \ldots, u_{n}\right)$ is a positive solution of (1.1) and $\left(k_{1}^{*} \varphi_{1}, \ldots, k_{n}^{*} \varphi_{1}\right)$ satisfy

$$
\left(\begin{array}{c}
\Delta k_{1}^{*} \varphi_{1} \\
\vdots \\
\Delta k_{n}^{*} \varphi_{1}
\end{array}\right)+\lambda_{*}\left(\begin{array}{ccc}
g_{11}^{\prime}(0) & \cdots & g_{n 1}^{\prime}(0) \\
\cdots & \ddots & \cdots \\
g_{1 n}^{\prime}(0) & \cdots & g_{n n}^{\prime}(0)
\end{array}\right)\left(\begin{array}{c}
k_{1}^{*} \varphi_{1} \\
\vdots \\
k_{n}^{*} \varphi_{1}
\end{array}\right)=0 .
$$

Multiplying the system (3.1) by $\left(k_{1}^{*} \varphi_{1}, k_{2}^{*} \varphi_{1}, \ldots, k_{n}^{*} \varphi_{n}\right)$, the system (3.13) by $\left(u_{1}, \ldots, u_{n}\right)$, integrating over $\Omega$ and subtracting, by $\left(\mathrm{A}_{2}\right)$ and $\sum_{j=1}^{n} g_{i j}(0)=0$, we obtain

$$
\begin{aligned}
\lambda_{*} \int_{\Omega} \sum_{i=1}^{n}\left[\sum_{j=1}^{n} k_{i}^{*} g_{i j}^{\prime}(0) u_{j} \varphi_{1}\right] d x & =\lambda \int_{\Omega} \sum_{j=1}^{n}\left[\sum_{i=1}^{n} k_{i}^{*} g_{i j}\left(u_{j}\right) \varphi_{1}\right] d x \\
& <\lambda \int_{\Omega} \sum_{j=1}^{n}\left[\sum_{i=1}^{n} k_{i}^{*} g_{i j}^{\prime}(0) u_{j} \varphi_{1}\right] d x .
\end{aligned}
$$

Hence (3.1) has no positive solution when $\lambda \leq \lambda_{*}$. And the solution $\left\{\left(\lambda(s), u_{1}(s), \ldots, u_{n}(s)\right)\right\}$ can also be parameterized as $\left(\lambda, u_{1}(\lambda), \ldots, u_{n}(\lambda)\right)$ for $\lambda \in\left(\lambda_{*}, \lambda_{*}+\delta\right)$. With the implicit function theorem, we can extend this curve to the largest $\lambda^{*}$.

Let $\Gamma=\left\{\left(\lambda, u_{1}(\lambda), \ldots, u_{n}(\lambda)\right): \lambda_{*}<\lambda<\lambda^{*}\right\}$. We show that $\left(u_{1}(\lambda), \ldots, u_{n}(\lambda)\right)$ is strictly increasing in $\lambda$ for $\lambda \in\left(\lambda_{*}, \lambda^{*}\right)$. In fact, $\left(\partial u_{1}(\lambda) / \partial \lambda, \ldots, \partial u_{n}(\lambda) / \partial \lambda\right)$ satisfies the equation

$$
\begin{aligned}
& F_{U}(\lambda, U)\left(\begin{array}{c}
\frac{\partial u_{1}(\lambda)}{\partial \lambda} \\
\vdots \\
\frac{\partial u_{n}(\lambda)}{\partial \lambda}
\end{array}\right) \\
& =\left(\begin{array}{c}
\Delta \frac{\partial u_{1}(\lambda)}{\partial \lambda} \\
\vdots \\
\Delta \frac{\partial u_{n}(\lambda)}{\partial \lambda}
\end{array}\right)+\lambda\left(\begin{array}{ccc}
g_{11}^{\prime}\left(u_{1}(\lambda)\right) & \cdots & g_{1 n}^{\prime}\left(u_{n}(\lambda)\right) \\
\vdots & \ddots & \vdots \\
g_{n 1}^{\prime}\left(u_{1}(\lambda)\right) & \cdots & g_{n n}^{\prime}\left(u_{n}(\lambda)\right)
\end{array}\right)\left(\begin{array}{c}
\frac{\partial u_{1}(\lambda)}{\partial \lambda} \\
\vdots \\
\frac{\partial u_{n}(\lambda)}{\partial \lambda}
\end{array}\right) \\
& =-\left(\begin{array}{c}
g_{11}\left(u_{1}(\lambda)\right)+\cdots+g_{1 n}\left(u_{n}(\lambda)\right) \\
\vdots \\
g_{n 1}\left(u_{1}(\lambda)\right)+\cdots+g_{n n}\left(u_{n}(\lambda)\right)
\end{array}\right) .
\end{aligned}
$$

Hence $\left(\partial u_{1}(\lambda) / \partial \lambda, \ldots, \partial u_{n}(\lambda) / \partial \lambda\right)$ from the maximum principle (Lemma 2.2 part 3$)$ and the fact that $\mu_{1}\left(\left(u_{1}(\lambda), \ldots, u_{n}(\lambda)\right)\right)>0$ from the stability of positive solutions. We claim that $\lambda^{*}=\infty$. Suppose not, then $\lambda^{*}<\infty$ and $\left\|\left(u_{1}(\lambda), \ldots, u_{n}(\lambda)\right)\right\|_{X}<\infty$. Then one can show that the curve $\Gamma$ can be extended to $\lambda=\lambda^{*}$ from some standard elliptic estimates, then from the implicit function theorem, $\Gamma$ can be extended beyond $\lambda=\lambda^{*}$, which is a contradiction; if $\lambda^{*}<\infty$ and $\left\|\left(u_{1}(\lambda), \ldots, u_{n}(\lambda)\right)\right\|_{X}=\infty$, a contradiction can be derived with the solution curve which cannot blow up at finite $\lambda^{*}$ (see similar arguments for the scalar equation in [25]). Hence we must have $\lambda^{*}=\infty$.

Finally, we claim the uniqueness. If there is another positive solution for some $\lambda>\lambda_{*}$, then the arguments above show that this solution also belongs to a solution curve defined for $\lambda \in\left(\lambda_{*}, \infty\right)$, and the solutions on the curve are increasing in $\lambda$, but the nonexistence of positive solutions for $\lambda<\lambda_{*}$ and the local bifurcation at $\lambda=\lambda_{*}$ exclude the possibility of 
another solution curve. Hence the positive solution is unique for all $\lambda>\lambda_{*}$. This completes the proof.

Example 3.3 We consider the following cyclic system:

$$
\begin{cases}\Delta u_{1}+\lambda f_{1}\left(u_{2}\right)=0, & x \in \Omega, \\ \Delta u_{2}+\lambda f_{2}\left(u_{3}\right)=0, & x \in \Omega, \\ \cdots & \\ \Delta u_{n}+\lambda f_{n}\left(u_{1}\right)=0, & x \in \Omega, \\ u_{1}(x)=\cdots=u_{n}(x)=0, & x \in \partial \Omega,\end{cases}
$$

where $f_{i}$ are smooth real functions defined on $\mathbf{R}_{+}$satisfying $\left(\mathrm{A}_{1}\right),\left(\mathrm{A}_{2}\right)$ and $\left(\mathrm{A}_{3}\right)$. Then Theorem 3.2 implies the existence and uniqueness of a positive solution of problem (3.16), and the bifurcation diagram is a single monotone solution curve. The $n$-dimensional cyclic positone and semipositone system was considered in [26] and [27]. They got the existence and multiplicity of positive solutions result for some combined sublinear condition by the method of sub-super solutions.

Example 3.4 Consider the general Lane-Emden system

$$
\begin{cases}\Delta u_{1}+\lambda b_{1}\left(a_{11}+u_{1}\right)^{p_{11}} \cdots\left(a_{1 n}+u_{n}\right)^{p_{1 n}}=0, & x \in \Omega, \\ \cdots & \\ \Delta u_{i}+\lambda b_{i}\left(a_{i 1}+u_{1}\right)^{p_{11}} \cdots\left(a_{i n}+u_{n}\right)^{p_{i n}}=0, & x \in \Omega, \\ \cdots & \\ \Delta u_{i}+\lambda b_{n}\left(a_{n 1}+u_{1}\right)^{p_{n 1}} \cdots\left(a_{n n}+u_{n}\right)^{p_{n n}}=0, & x \in \Omega, \\ u_{1}(x)=\cdots=u_{n}(x)=0, & x \in \partial \Omega,\end{cases}
$$

where $\lambda>0$ is a positive parameter, $b_{i}, a_{i j}(1 \leq i, j \leq n)$ are positive constants, $p_{i j}$ ( $1 \leq$ $i, j \leq n)$ are nonnegative constants satisfying $\sum_{i=1}^{n} p_{i j}<1$ and $\Omega \subset \mathbf{R}^{N}$ denotes a bounded domain of class $C^{2, \alpha}, \alpha \in(0,1)$. For the case $N=2$, (3.17) has been studied by many authors. Especially, Dalmasso [10] proved the uniqueness and existence of positive solutions to (3.17) for the case $N=2, p_{11}=p_{22}=0,0<p_{12} p_{21}<1$.

In this section, we show the uniqueness and existence of positive solutions for (3.17) by using super-subsolution methods and the stability of positive solutions by using Theorem 2.4.

Let $e(x)$ be the unique positive solution of

$$
\Delta e+1=0, \quad x \in \Omega, \quad e(x)=0, \quad x \in \partial \Omega .
$$

We construct a super-solution $\left(\bar{u}_{1}, \ldots, \bar{u}_{n}\right)=M(e(x), \ldots, e(x))$. There exists a suitable positive constant $M$ such that

$$
\begin{gathered}
\Delta(M e)+\lambda b_{i}\left(a_{i 1}+M e\right)^{p_{i 1}} \cdots\left(a_{i n}+M e\right)^{p_{i n}} \\
\leq-M+\lambda b_{i}\left(\bar{a}_{i}+M e\right)^{p_{11}+\cdots+p_{i n}} \leq 0,
\end{gathered}
$$


where $\bar{a}_{i}=\max \left\{a_{i 1}, \ldots, a_{i n}\right\}$. We construct a sub-solution in the form of $\left(\underline{u}_{1}, \ldots, \underline{u}_{n}\right)=$ $\left(\varepsilon \varphi_{1}, \ldots, \varepsilon \varphi_{1}\right)$, where $\varepsilon$ will be specified later. Recall that $\varphi_{1}$ is the positive principal eigenfunction with $\left\|\varphi_{1}\right\|_{\infty}=1$. Now, for the $i$ th equation of (3.17), we have

$$
\begin{aligned}
\Delta\left(\varepsilon \varphi_{1}\right)+\lambda b_{i}\left(a_{i 1}+\varepsilon \varphi_{1}\right)^{p_{i 1}} \cdots\left(a_{i n}+\varepsilon \varphi_{1}\right)^{p_{i n}} & \geq-\varepsilon \lambda_{1} \varphi_{1}+\lambda b_{i}\left(\underline{a}_{i}+\varepsilon \varphi_{1}\right)^{p_{i 1}+\cdots+p_{i n}} \\
& \geq-\varepsilon \lambda_{1} \varphi_{1}+\lambda b_{i}\left(\varepsilon \varphi_{1}\right)^{p_{11}+\cdots+p_{i n}}
\end{aligned}
$$

where $\underline{a}_{i}=\min \left\{a_{i 1}, \ldots, a_{i n}\right\}$. Hence $\left(\underline{u}_{1}, \ldots, \underline{u}_{n}\right)$ is a subsolution of (3.17), if we choose $\varepsilon$ smaller, and it satisfies $\varepsilon<\left(\lambda b_{i} / \lambda_{1}\right)^{\frac{1}{1-\left(p_{i 1}+\cdots+p_{i n}\right)}}\left\|\varphi_{1}\right\|_{\infty}^{-1}$. Hence if we choose $\varepsilon$ smaller so that $\left(\underline{u}_{1}, \ldots, \underline{u}_{n}\right) \leq\left(\bar{u}_{1}, \ldots, \bar{u}_{n}\right)$, then $(\underline{u}, \underline{v})$ is a subsolution of $(4.1)$. Therefore, there exists a positive solution $\left(u_{1}, \ldots, u_{n}\right)$ of (3.17) between the supersolution and the subsolution.

Next, we show the solution is stable. Letting $f_{i}(U)=b_{i} \prod_{j=1}^{n}\left(a_{i j}+u_{j}\right)^{p_{i j}}$, by simple calculation, we get

$$
f_{i j}^{\prime}(U)=b_{i} p_{i j} \prod_{l=1, l \neq j}^{n}\left(a_{i l}+u_{l}\right)^{p_{i l}}\left(a_{i j}+u_{i j}\right)^{p_{i j}-1}
$$

and

$$
\begin{aligned}
f_{i}(U)-\sum_{j=1}^{n} f_{i j}^{\prime}(U) u_{j} & =b_{i} \prod_{j=1}^{n}\left(a_{i j}+u_{j}\right)^{p_{i j}}-\sum_{j=1}^{n} b_{i} p_{i j} \prod_{l=1, l \neq j}^{n}\left(a_{i l}+u_{l}\right)^{p_{i l}}\left(a_{i j}+u_{j}\right)^{p_{i j}-1} \\
& =b_{i} \prod_{l=1}^{n}\left(a_{i l}+u_{l}\right)^{p_{i l}}\left[1-\sum_{j=1}^{n} \frac{p_{i j}}{a_{i j}+u_{j}} u_{j}\right] \\
& >b_{i} \prod_{l=1}^{n}\left(a_{i l}+u_{l}\right)^{p_{i l}}\left[1-\sum_{j=1}^{n} p_{i j}\right]>0 .
\end{aligned}
$$

So, $f_{i}$ satisfies $\left(\mathrm{H}_{1}\right)$, hence from Theorem 2.4 , any positive solution of (3.17) is stable.

\section{Application: Hölder continuous case}

In this section, we consider that $f_{i} \in C^{\alpha}\left(\overline{\mathbf{R}}_{+}^{n}\right) \cap C^{1}\left(\mathbf{R}_{+}^{n}\right)$ for $\alpha \in(0,1)$.

Example 4.1 Consider

$$
\begin{cases}\Delta u+\lambda\left(a u-u^{2}+\sqrt{u v}\right)=0, & x \in \Omega, \\ \Delta v+\lambda\left(b v-v^{2}+\sqrt{u v}\right)=0, & x \in \Omega, \\ u(x)=v(x)=0, & x \in \partial \Omega,\end{cases}
$$

where $\lambda>0$ is a positive parameter, $a, b \geq 0$.

We use the monotone method to prove the existence of a solution. For the supersolution, we choose $(\bar{u}, \bar{v})=(a+b+1, a+b+1)$. Then

$$
\begin{aligned}
\Delta \bar{u}+\lambda\left(a \bar{u}-\bar{u}^{2}+\sqrt{\overline{u v}}\right) & \leq \lambda\left[a(a+b+1)-(a+b+1)^{2}+(a+b+1)\right] \\
& \leq \lambda(a+b+1)[(a+1)-(a+b+1)] \leq 0 .
\end{aligned}
$$


Similarly, we have $0<v<(a+b+1)$. Then it is clear that $(\bar{u}, \bar{v})$ is a supersolution of (4.1). We construct a sub-solution in the form of $(\underline{u}, \underline{v})=\left(\varepsilon \varphi_{1}, \varepsilon \varphi_{1}\right)$, where $\varepsilon$ will be specified later. Recall that $\varphi_{1}$ is the positive principal eigenfunction with $\left\|\varphi_{1}\right\|_{\infty}=1$. Now, for the equation of $u$ or the equation of $v$, we have

$$
\begin{aligned}
\Delta\left(\varepsilon \varphi_{1}\right)+\lambda\left(a \varepsilon \varphi_{1}-\left(\varepsilon \varphi_{1}\right)^{2}+\varepsilon \varphi_{1}\right) & =-\varepsilon \lambda_{1} \varphi_{1}+\lambda\left(a \varepsilon \varphi_{1}-\left(\varepsilon \varphi_{1}\right)^{2}+\varepsilon \varphi_{1}\right) \\
& =\varepsilon \varphi_{1}\left[\lambda(a+1)-\lambda_{1}-\lambda \varepsilon \varphi_{1}\right] .
\end{aligned}
$$

Hence if we choose $\varepsilon$ smaller and it satisfies $\varepsilon<\min \left\{\frac{\lambda(a+1)-\lambda_{1}}{\lambda\left\|\varphi_{1}\right\|_{\infty}}, \frac{\lambda(b+1)-\lambda_{1}}{\lambda\left\|\varphi_{1}\right\|_{\infty}}\right\}$, so that $(\underline{u}, \underline{v}) \leq$ $(\bar{u}, \bar{v})$, then $(\underline{u}, \underline{v})$ is a subsolution of (4.1). Therefore, there exists a positive solution $(u, v)$ of (4.1) between the supersolution and the subsolution when $\lambda>\max \left\{\lambda_{1} /(a+1), \lambda_{1} /(b+1)\right\}$. We remark that the stability defined in Section 2 can still be established for a nonlinear function $f_{i}^{\prime}$ to become $\infty$ near $\partial \Omega$ by using Remark 3.1 in [28]. Thus the positive solution $(u, v)$ of $(4.1)$ is stable.

Remark 4.2 Since (4.1) is a cooperative model from ecology with logistic growth rate and sublinear interaction term, we can get the stable result. When the interaction terms are $u v$ (Lotka-Volterra type) and $\sqrt{u v}$ as proposed here, and they do not satisfy the conditions of Theorem 3.2, thus, the solution may not be unique.

Competing interests

The authors declare that they have no competing interests.

\section{Authors' contributions}

BW and RC carried out the proof of the main part of this article, RC corrected the manuscript and participated in its design and coordination. All authors have read and approved the final manuscript.

\footnotetext{
Acknowledgements

Partially supported by the National Natural Science Foundation of China (No. 11071051, 11271100 and 11101110), the Aerospace Supported Fund, China, under Contract (Grant 2011-HT-HGD-06), Science Research Foundation of the Education Department of Heilongjiang Province (Grant No. 12521153), Science Foundation of Heilongjiang Province (Grant A201009) and Harbin Normal University advanced research Foundation (11xyg-02).
}

Received: 19 December 2012 Accepted: 14 March 2013 Published: 4 April 2013

\section{References}

1. Chen, ZY, Chern, JL, Shi, J, Tang, YL: On the uniqueness and structure of solutions to a coupled elliptic system. J. Differ. Equ. 249, 3419-3442 (2010)

2. Chern, JL, Tang, YL, Lin, CS, Shi, J: Existence, uniqueness and stability of positive solutions to sublinear elliptic systems. Proc. R. Soc. Edinb. A 141, 45-64 (2011)

3. Cui, RH, Li, P, Shi, J, Wang, YW: Existence, uniqueness and stability of positive solutions for a class of semilinear elliptic systems. Topol. Methods Nonlinear Anal. (to appear)

4. Shi, J, Shivaji, R: Exact multiplicity of positive solutions to cooperative elliptic systems. Preprint (2012)

5. Korman, P, Li, Y, Ouyang, T: Exact multiplicity results for boundary value problems with nonlinearities generalising cubic. Proc. R. Soc. Edinb. A 126, 599-616 (1996)

6. Korman, $\mathrm{P}, \mathrm{Li}, \mathrm{Y}, \mathrm{Ouyang}, \mathrm{T}$ : An exact multiplicity result for a class of semilinear equations. Commun. Partial Differ. Equ. 22, 661-684 (1997)

7. Ouyang, T, Shi, J: Exact multiplicity of positive solutions for a class of semilinear problem. J. Differ. Equ. 146, 121-156 (1998)

8. Ouyang, T, Shi, J: Exact multiplicity of positive solutions for a class of semilinear problem, II. J. Differ. Equ. 158, 94-151 (1999)

9. Sirakov, B: Notions of sublinearity and superlinearity for nonvariational elliptic systems. Discrete Contin. Dyn. Syst. 13, 163-174 (2005)

10. Dalmasso, R: Existence and uniqueness of positive solutions of semilinear elliptic systems. Nonlinear Anal. 39 559-568 (2000)

11. Hai, DD: Existence and uniqueness of solutions for quasilinear elliptic systems. Proc. Am. Math. Soc. 133, 223-228 (2005)

12. Hai, DD: Uniqueness of positive solutions for semilinear elliptic systems. J. Math. Anal. Appl. 313, 761-767 (2006) 
13. Hai, DD, Shivaji, R: An existence result on positive solutions for a class of semilinear elliptic systems. Proc. R. Soc. Edinb. A 134, 137-141 (2004)

14. Troy, WC: Symmetry properties in systems of semilinear elliptic equations. J. Differ. Equ. 42, 400-413 (1981)

15. Serrin, J, Zou, H: Existence of positive solutions of the Lane-Emden system. Atti Semin. Mat. Fis. Univ. Modena 46, suppl. 369-380 (1998)

16. Serrin, J, Zou, H: Existence of positive entire solutions of elliptic Hamiltonian systems. Commun. Partial Differ. Equ. 23, 577-599 (1998)

17. Korman, P, Shi, J: On Lane-Emden type systems. Discrete Contin. Dyn. Syst. suppl., 510-517 (2005)

18. Cui, RH, Wang, YW, Shi, J: Uniqueness of the positive solution for a class of semilinear elliptic systems. Nonlinear Anal. 67, 1710-1714 (2007)

19. Cui, RH, Shi, J, Wang, YW: Existence and uniqueness of positive solutions for a class of semilinear elliptic systems. Acta Math. Sin. Engl. Ser. 27, 1079-1090 (2011)

20. Masaaki, M: Uniqueness and existence of positive solutions for some semilinear elliptic systems. Nonlinear Anal. 59 993-999 (2004)

21. Sweers, G: Strong positivity in $C(\bar{\Omega})$ for elliptic systems. Math. Z. 209, 251-271 (1992)

22. Kato, T: Perturbation Theory for Linear Operators. Reprint of the 1980 edition. Classics in Mathematics. Springer, Berlin (1995)

23. Serre, D: Matrices: Theory and Applications. Graduate Texts in Mathematics, vol. 216. Springer, Berlin (2002)

24. Crandall, MG, Rabinowitz, PH: Bifurcation from simple eigenvalues. J. Funct. Anal. 8, 321-340 (1971)

25. Shi, J: Blow up points of solution curves for a semilinear problem. Topol. Methods Nonlinear Anal. 15, 251-266 (2000)

26. Ali, J, Brown, K, Shivaji, R: Positive solutions for $n \times n$ elliptic systems with combined nonlinear effects. Differ. Integral Equ. 24(3-4), 307-324 (2011)

27. Lee, EK, Shivaji, R, Ye, J: Classes of infinite semipositone $n \times n$ systems. Differ. Integral Equ. 24(3-4), 361-370 (2011)

28. Ambrosetti, A, Brézis, H, Cerami, G: Combined effects of concave and convex nonlinearities in some elliptic problems. J. Funct. Anal. 122, 519-543 (1994)

doi:10.1186/1687-2770-2013-74

Cite this article as: Wu and Cui: Existence, uniqueness and stability of positive solutions to a general sublinear elliptic systems. Boundary Value Problems 2013 2013:74.

\section{Submit your manuscript to a SpringerOpen ${ }^{\circ}$ journal and benefit from:}

- Convenient online submission

Rigorous peer review

- Immediate publication on acceptance

- Open access: articles freely available online

- High visibility within the field

- Retaining the copyright to your article 